\title{
Estrategias pedagógicas para el aprendizaje del inglés con el uso de la herramienta Rosseta Stone por parte de los estudiantes de grado octavo del Colegio León de Greiff
}

Rosa Lidya Rangel Palencia ${ }^{1}$

\begin{abstract}
Resumen
Las posibilidades del ciberespacio en el mundo educativo van más allá de sus aportes como medio de información o fuente de recursos didácticos y comunicativos. Permite a los estudiantes desarrollar capacidades y tomar conciencia de sus opiniones formadas a partir de la reflexión crítica y la autonomía. El acceso al ciberespacio posibilita la apropiación de contenido y la construcción conjunta del mismo en colaboración con otros usuarios. En este sentido, la relación entre tecnología y educación se hace más patente y necesaria.
\end{abstract}

Este artículo de carácter reflexivo, parte de una investigación cualitativa de tipo hermenéutico, presenta los resultados, producto de los procesos de interactividad de los estudiantes de grado octavo del Colegio León de Greiff, del Municipio de Aguazul, con la web Rosseta Stone. A través de dicho análisis se evidencia la potencialización del idioma inglés con dicha web y se formulan algunas estrategias pedagógicas que permiten el aprovechamiento del ciberespacio dentro y fuera del aula de clase.

Palabras clave: aprendizaje del inglés, cibercultura, ciberespacio, comunicación, educación. 


\title{
Teaching strategies for learning English using the Rosetta Stone tool by eighth graders in the Leon de Greiff College
}

\begin{abstract}
The possibilities of cyberspace in the educational world beyond their contributions as an information source or didactic and communication resources. It allows students to develop skills and become aware of their opinions formed from critical reflection and autonomy. Access to cyberspace enables content ownership and joint construction of it in collaboration with others. In this sense, the relationship between technology and education is more evident and necessary.

This article reflective nature of qualitative research hermeneutical kind, presents the results, due to the processes of interaction of the eighth grade students of the León de Greiff School, Aguazul Township, with web Rosetta Stone. Through this analysis the potentiation of the English language is evidence that site and some teaching strategies that allow the use of cyberspace inside and outside the classroom are formulated.
\end{abstract}

Keywords: Cyberspace, Education, Communication, Cyberculture, English Language.

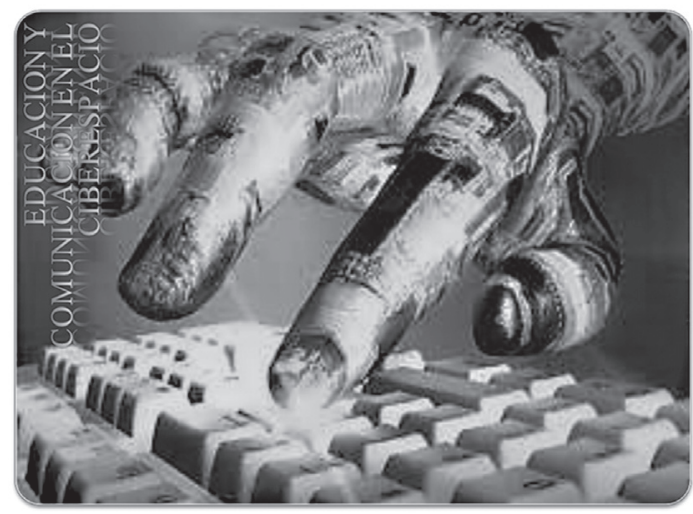

Tomado de: www.imagenesgoogle.com

La inteligencia consiste no sólo en el conocimiento, sino también en la destreza de aplicar los conocimientos en la práctica. 


\section{Introducción}

En el proceso de globalización, las tecnologías de la información y la comunicación (TIC), han generado entre los seres humanos una gran revolución en la manera de comunicarse, relacionarse, acceder, apropiarse y transmitir la información, lo que ha hecho surgir nuevos cambios en los diferentes ámbitos de la sociedad, en especial, en el campo educativo. Los cambios que propician las TIC, se deben en gran medida a las características que presentan, como son: el fácil acceso y procesamiento de todo tipo de información, la comunicación inmediata, ya sea sincrónica o a sincrónica, la posibilidad de almacenar grandes cantidades de información y la interactividad posible entre ordenadores o usuarios.

En el marco de la Revolución Educativa Colombiana, el programa vincula el uso de medios y nuevas tecnologías de información y comunicación para optimizar la enseñanza y el aprendizaje, reducir la brecha digital y aprovechar las ventajas de la conectividad y las nuevas formas de alfabetización. Mediante el uso de tecnologías y medios es posible llegar a más personas, sin grandes desplazamientos y reduciendo costos, bien sea con la televisión educativa, la radio, los medios impresos o la conexión a la red de redes (MEN, 2005: 3).

En septiembre del año 2013, el British Council y el Programa Computadores para Educar del Ministerio de Tecnologías de la Información y las Comunicaciones MinTIC, firmaron un acuerdo de cooperación en Tecnología para la Educación. A través de este proyecto conjunto el British Council pone a disposición de estudiantes y docentes contenidos digitales para facilitar los procesos de aprendizaje del inglés en zonas urbanas y rurales de toda Colombia. Los contenidos digitales fueron instalados en computadores portátiles del Programa Computadores para Educar del MinTIC e incluyen audio, videos, un compendio de explicaciones gramaticales, animaciones interactivas en flash y archivos en formato PDF que se pueden imprimir y fotocopiar. Estos contenidos están clasificados en once categorías generales y forman parte de «Learn English»; página web disponible en http://learnenglish.britishcouncil.org/en/, desarrollada por el British Council para la enseñanza y el aprendizaje del inglés.

Así mismo, el Ministerio de Educación Nacional, en el marco de la política sectorial Educación de Calidad el Camino para la Prosperidad, ha puesto en marcha el Proyecto de Fortalecimiento al Desarrollo de Competencias en Lenguas Extranjeras, cuyo objetivo es «desarrollar competencias comunicativas en lenguas extranjeras, particularmente el inglés, en educadores y estudiantes del sector oficial para favorecer la inserción del capital humano colombiano a la economía del conocimiento y un mercado laboral globalizado» (2010: 6).

Es por ello que hoy en día, el aprendizaje de otros idiomas y la comprensión de la cultura de sus hablantes constituyen habilidades esenciales para el desarrollo y 
el éxito de los estudiantes en el mundo globalizado del siglo XXI. Aprender otra lengua no solo contribuye al desarrollo cognitivo y profesional, sino también a respetar y comprender mejor la visión de mundo y de otras culturas. En éste sentido, saber otros idiomas es fundamental para poder interactuar en forma efectiva con el resto del mundo y acceder a nuevos conocimientos a través de los medios tecnológicos y las redes de comunicación social.

Es así como el Ministerio de Educación Nacional formula el Programa Nacional de Bilingüismo 2004-2019, que incluye los nuevos estándares de competencias comunicativas en lengua extranjera inglés. Con el Marco Común Europeo como referente nacional e internacional, el programa se propone elevar la competencia comunicativa en inglés en todo el sistema educativo y fortalecer la competitividad nacional. En esta tarea juegan un papel decisivo los docentes y las instituciones educativas, públicas y privadas, y todos los niveles que hacen parte del sistema, desde el Preescolar hasta el Superior (MEN, 2006: 3).

Ser bilingüe es esencial en un mundo globalizado. El manejo de una segunda lengua permite una mejor comunicación, abrir fronteras, comprender otros contextos, apropiar saberes y hacerlos circular, entender y hacernos entender, enriquecerse y jugar un papel decisivo en el desarrollo del país. Ser bilingüe es tener más conocimientos y oportunidades para ser más competentes y competitivos, y mejorar la calidad de vida de todos los ciudadanos.

La Ley 115 de 1994 en sus objetivos para la educación Básica y Media, demanda «la adquisición de elementos de conversación, lectura, comprensión y capacidad de expresarse al menos en una lengua extranjera». A partir de su promulgación, la mayoría de las instituciones escolares adoptan la enseñanza del inglés como lengua extranjera. De ahí el compromiso del Ministerio de Educación con la creación de condiciones para apoyar a las Secretarías de Educación e involucrar en el proceso a todos los implicados en la enseñanza, el aprendizaje y la evaluación de esa lengua, universidades, centros de lengua, organismos de cooperación internacional y proveedores de materiales educativos, entre otros (Ley 115 de 1994, art 21, 22, 23, 27, 30).

La presente propuesta de investigación se orientó hacia el fortalecimiento del aprendizaje del idioma inglés a través de la web Rosseta Stone, la cual está comprendida en la línea de Investigación de Ambientes Virtuales, E-learning y Educación de la Universidad Santo Tomás. Esta investigación parte de un enfoque cualitativo de tipo hermenéutico, buscando describir y caracterizar los procesos de interactividad producidos por los estudiantes de grado octavo del Colegio León de Greiff, del municipio de Aguazul, en el sitio web Rosseta Stone. A través del análisis de dichas interacciones con la web expuesta, se formula una serie de estrategias pedagógicas que permitan el aprovechamiento del ciberespacio dentro y fuera del aula de clase. 
La institución educativa León de Greiff es una institución de carácter rural ubicada en el departamento de Casanare y cerca a uno de los pozos petroleros más importantes del país. Esta investigación surge de la motivación y la necesidad de formar y mejorar la competencia comunicativa del idioma inglés de la población local. Así como de articular dichos conocimientos con las tecnologías existentes en el contexto educativo, con el fin de que los estudiantes se enfrenten de manera exitosa a las exigencias laborales y educativas de la región y del país.

Por lo tanto, el proceso investigativo tuvo como eje principal la población de estudiantes y docentes de educación básica, buscando aprovechar pedagógicamente dicho medio en la potencialización del aprendizaje del idioma inglés. De ésta manera, esta propuesta busca que la población educativa comprenda que el ciberespacio enriquece en los planos personal, intelectual, espiritual y lexical; siempre y cuando haya un sentido de responsabilidad en cuanto a dicha herramienta y un acompañamiento adecuado por parte de un docente, tutor o guía.

Francisco García (2004), señala como hoy en día se requiere que los docentes desarrollen una tarea fundamental «Educar en el ciberespacio» (p. 4). Pero, educar para Internet y con Internet conlleva cambios en el rol del profesor, pasando del profesor transmisor de conocimiento en la educación tradicional al de orientador, facilitador, colaborador, guía del proceso de aprendizaje de cada estudiante de forma permanente, llegando a disponer a cualquier hora, en cualquier momento y en cualquier lugar de un canal de comunicación y de interacción con sus iguales y con el resto de agentes educativos, con la finalidad de desarrollar las habilidades y competencias informativas e informativas individuales, para aprender a aprender, aprender a conocer, aprender a hacer y aprender a comprender al otro manteniendo una actitud crítica (García, 2004: 4).

La investigación se enmarca dentro de la metodología de investigación cualitativa de tipo hermenéutico, a partir de la cual se describen y caracterizan los procesos de interactividad producidos por los estudiantes de grado octavo del Colegio León de Greiff, del municipio de Aguazul, en el sitio web Rosseta Stone. Plantea como pregunta investigativa ¿Cuáles son los procesos de interactividad producidos por los estudiantes de grado octavo del Colegio León de Greiff, del municipio de Aguazul, en el sitio web Rosseta Stone, como mediación para potencializar el aprendizaje del inglés? Los resultados que sobresalen evidencian como el ciberespacio es una herramienta pedagógica que potencializa el aprendizaje del idioma inglés, siempre y cuando exista una responsabilidad, tanto en el rol del docente-tutor y los estudiantes en la realización de cada una de las actividades on-line y off-line con pertinencia, autonomía, y con su respectiva retroalimentación. 


\section{Metodología}

La presente investigación se enmarco dentro del tipo de investigación cualitativa. Se estructuró a partir de varias etapas. La primera involucró la recolección de información haciendo uso de una encuesta para percibir el grado de apropiación del ciberespacio por parte de los estudiantes de grado octavo del Colegio León de Greiff, que tienen acceso a la Sala de Bilingüismo de la institución y se empezó por clasificar las diferentes instancias del uso, acceso y dificultades que los estudiantes pudiesen tener al momento de utilizar la web Rosseta Stone, en relación con el aprendizaje del inglés. Luego, se diseñaron unas rejillas de observación para registrar, observar, describir, analizar y caracterizar cada una de las interacciones de los estudiantes con la web Rosseta Stone, finalizando con el análisis de resultados y las conclusiones que permiten plantear estrategias pedagógicas que permiten el aprovechamiento del ciberespacio dentro y fuera del aula de clase.

El método que delineó el proceso investigativo es la etnografía virtual, como un método clásico de la investigación cualitativa que permite al investigador estudiar de manera directa el comportamiento de un grupo de personas, durante un periodo de tiempo, gracias al trabajo de campo realizado a través de diversas técnicas como por ejemplo entrevistas, observación, historias de vida, el método genealógico, entre otros.

Christine Hine (2004), define la etnografía virtual como «una etnografía de Internet puede observar con detalle las formas en que se experimenta el uso de una tecnología. En su forma básica, la etnografía consiste en que un investigador se sumerja en el mundo que estudia por un tiempo determinado y tome en cuenta las relaciones, actividades y significaciones que se forjan entre quienes participan en los procesos sociales de ese mundo» (p. 13). Por lo tanto, una etnografía del ciberespacio observa las formas en que se experimenta el uso de la tecnología, el investigador se sumerge en un mundo de relaciones, actividades y significaciones que se visualizan a través del lenguaje virtual.

\section{Sistema teórico}

El sistema teórico se plantea a partir de las categorías centrales del problema de investigación, las cuales se desarrollan a continuación.

\section{El ciberespacio}

El ciberespacio ofrece un sin número de oportunidades para que el individuo pueda afianzar su aprendizaje de forma autónoma para satisfacer las competencias que demanda la sociedad globalizada. Es por ello que el uso de éste en la enseñanza constituye un recurso mediador del aprendizaje y de motivación para practicar 
y usar el idioma en situaciones reales, además de ayudar en la mejora de las habilidades lingüísticas de una manera interactiva y colaborativa.

El ciberespacio como una de las tecnologías emergentes en el siglo XXI, ha venido incrementando su uso en los últimos años en el área de la enseñanza de los idiomas con el propósito de trascender el aula de clase y promover la autonomía y autorregulación del aprendizaje por parte de los estudiantes. Esta nueva concepción de la web se ajusta a los objetivos que se persiguen en la enseñanza y aprendizaje de lenguas, como son la presentación de un contenido dinámico, facilidades para la creatividad, la autonomía, la interacción, la colaboración, las comunidades virtuales y el uso de la lengua en contextos auténticos y reales, los cuales favorecen la interactividad y la colaboración entre los estudiantes; además, permite el acceso y difusión del conocimiento más allá del aula tradicional potenciando de esta manera el aprendizaje.

Algunas de las plataformas presentadas en la web, entre las que cabe destacar, Pumarosa, Hot-potatoes, Educaplay, La mansión del inglés, Duolingo, Livemocha, Yellow-pencil y por supuesto Rosseta Stone, entre otras, facilitan al estudiante de inglés el contacto directo con profesores y hablantes del idioma, además de funcionar como un medio auténtico, conveniente y de alta accesibilidad para publicaciones multimedia en dicho idioma. Esto conduce a que los estudiantes creen su propio contenido y construyan su identidad en línea a través de espacios interactivos donde puedan reflexionar y usar el idioma con sus compañeros, sobre la base de una comunicación virtual que los conecta con el mundo globalizado de hoy.

El ciberespacio es una realidad simulada que se encuentra implementada dentro de los ordenadores y de las redes digitales de todo el mundo. Se ha convertido en el medio más popular para la divulgación de la información, que ha influido en todas las actividades sociales de la humanidad, incluyendo en la forma de interacción entre las personas. Esta forma de difusión de la información proporciona grandes ventajas sobre otros medios de difusión, como por ejemplo, poder difundir información a diferentes personas ubicadas en diferentes y variados sitios geográficos, con la posibilidad de proveer textos, sonidos e imágenes.

En el ámbito educativo, el ciberespacio ofrece ciertas ventajas con respecto a la educación tradicional que hacen que este medio sea muy atractivo tanto para los facilitadores de aprendizaje (profesores), como para los usuarios (estudiantes) de este tipo de recursos, ya que permite a los facilitadores poner a disposición de los usuarios, materiales educativos que puedan ser utilizados como complemento a su formación, entre otros. Por lo tanto, los múltiples caminos abiertos por las nuevas tecnologías, no sólo le han aportado al hombre diversas posibilidades para interactuar en el mundo, también le han permitido acceder y vincularse a espacios más dinámicos y virtuales, donde día a día nuevos códigos se articulan para generar redes ilimitadas de significación e interpretación. 
Levy (1999), señala que «el ciberespacio constituye un soporte para las tecnologías intelectuales que amplifican, exteriorizan y modifican numerosas funciones cognitivas del ser humano: la memoria, la imaginación, la percepción, los razonamientos. Estas tecnologías intelectuales favorecen nuevas formas de acceso a la información y nuevos estilos de razonamiento y conocimiento» ( $\mathrm{p}$. 7). Así mismo Lévy (1999: 7), presenta las implicaciones del ciberespacio para la educación en tres niveles:

a) Nuevas formas de acceso a la información. El ciberespacio proporciona acceso en tiempo real a los distintos recursos provenientes de todos los lugares del planeta. Los estudiantes pueden participar en conferencias electrónicas, la red favorece la accesibilidad, en un doble sentido: para la producción y para el consumo. Esto conduce a que el conocimiento presente en el ciberespacio sea no cuantificable e inabarcable, los conocimientos emergen constantemente, lo cual hace posible y necesaria la permanente actualización de los mismos. Es posible acceder sin restricciones y de forma directa a la información de última hora y a los discursos de los especialistas.

b) Nuevos estilos de razonamiento y conocimiento: el paradigma deja de ser «el curso» y pasa a ser «la navegación». En el ciberespacio se accede al conocimiento de manera a la vez masiva y personalizada. El ciberespacio posibilita el aprendizaje cooperativo, éste constituye para Lévy la tendencia más prometedora en lo que concierne a los nuevos modelos de adquisición de conocimientos. El ciberespacio promueve la inteligencia colectiva y se alimenta de ella.

c) Formas de organización social: Posibilidad de una comunicación directa e interactiva. La interconexión favorece los procesos de inteligencia colectiva en las comunidades virtuales. En el ciberespacio es posible compartir los recursos materiales e informáticos sin importar el tiempo y la distancia.

\section{La interactividad}

Chen (2001: 460), señala cuatro interacciones transaccionales esenciales; la primera interacción es aprendiz-instructor, la cual implica las posibilidades del aprendiz de apropiarse de nuevos conceptos gracias al acompañamiento en la explicación y aplicación de los mismos que realiza el instructor, a partir de los conocimientos previos (los conceptos que ya tenía apropiados) del aprendiz que disminuye la distancia transaccional: transacción (negociación) entre el proceso comunicativo (social) y la apropiación (correlación con los conceptos previos, internalización). 
Se trata de la interacción del estudiante con el tutor o docente, en este punto, el rol del docente es el de «suscitar integralmente la potencia deliberativa de sus estudiantes» (Chen, 2001: 460), motivarlos para el aprendizaje autónomo, entendido como el proceso intelectual mediante el cual se ponen en ejecución estrategias cognitivas conscientes y secuenciales. Este proceso está regido por principios de acción, tales como: interés manifiesto que motive la actuación deliberada; reconocimiento de experiencias de aprendizaje previas, y trabajo cooperativo y colaborativo apoyado por actividades interconectadas e independientes.

Chen señala que la siguiente interacción se observa entre aprendiz-aprendiz; es la «distancia psicológica» que los aprendices perciben mientras que obran recíprocamente con otros aprendices. Se trata de la interacción de los mismos estudiantes. Las funciones que cumple van desde las sociales hasta las de comparación de grupo, todo aprendizaje implica la cooperación de los demás (Chen, 2001: 460). El aula virtual, con sus posibilidades de creación de campus, de actividades de grupo, facilitadora de la sensación de pertenencia a una comunidad de estudiantes, es la base para el trabajo cooperativo y colaborativo.

Como tercera interacción, Chen (2001), expone la interacción entre aprendizcontenido, definida como la comprensión que los aprendices perciben al estudiar los materiales del curso y el grado que los materiales resuelven sus necesidades y expectativas de aprendizaje. En éste mismo sentido, Holmberg (1986), señala que la interactividad aprendiz-contenido trata de la interacción de quien estudia y el material educativo que se pone a su disposición y otros materiales necesarios que ayuden a consolidar sus conocimientos, como lecturas obligatorias y consultas en la red, los cuales sirven de apoyo a los insumos básicos dispuestos por la institución educativa. Es por tanto el proceso de interacción intelectual con el contenido o los insumos básicos, que provocará cambios en las estructuras cognoscitivas del sujeto, producto de un tipo de conversación didáctica interna.

Por último, el autor presenta la interacción entre aprendiz-interfaz, relacionada en el cómo los aprendices perciben el uso fácil o difícil de los sistemas informático que median este aprendizaje. Comprende desde el manejo de la plataforma educativa, el uso adecuado y eficiente de las herramientas virtuales, la navegación por internet, hasta las normas de presentación de los trabajos. Incluye también el desarrollo de competencias comunicativas al redactar correos y la forma de expresar sus ideas y argumentos en un chat, sin olvidar las normas de netiqueta. En esta propuesta, un elemento clave es la ubicación del aprendiz como centro de los diferentes tipos de Interacciones que se muestran, y el presentar diferentes elementos del Sistema de Actividad: comunidad, roles, instrumentos (Chen, 2001: 461). 


\section{El aprendizaje del inglés desde el enfoque comunicativo}

Desde hace ya más de veinte años, el enfoque predominante en didáctica de segundas lenguas y de lenguas extranjeras es el enfoque comunicativo. Una enseñanza de tipo comunicativo es aquella que se marca como objetivo el desarrollo de la competencia comunicativa. La esencia de este enfoque se encuentra, pues, en el enriquecimiento de los objetivos de aprendizaje: no se trata únicamente de que los estudiantes adquieran un determinado sistema lingüístico, sino de que sean capaces de utilizarlo para comunicarse de forma adecuada y efectiva.

En el enfoque comunicativo el desarrollo de la competencia comunicativa representa el fin a alcanzar en el proceso de enseñanza y aprendizaje de la lengua. Para llegar a ella es preciso desarrollar, según Hymes, «un conjunto de procesos y conocimientos de diversos tipos (lingüísticos, discursivos, sociolingüísticos, socioculturales y estratégicos) que el hablante-oyente-escritor-lector deberá poner en juego para producir o comprender discursos adecuados a la situación y el contexto de comunicación y al grado de formalización requerido» (Hymes, 1966: 15).

En relación con el enfoque comunicativo, Nunan (1991: 25), resalta el énfasis en aprender a comunicarse a través de la interacción en el idioma extranjero, la introducción de materiales auténticos en la situación de aprendizaje, las oportunidades para centrarse no sólo en el idioma, sino en el propio proceso de aprendizaje, el incremento de las propias experiencias personales como elementos importantes que contribuyen al aprendizaje, así como el intento por vincular el aprendizaje del idioma en el aula con actividades en el idioma fuera del aula.

El contexto de la enseñanza de idiomas, por su carácter dialógico e interactivo, propicia el desarrollo de la competencia intercultural más compleja y de mayor valor educativo. En este sentido García y Granados (1999), la definen como «el proceso por el que una persona desarrolla competencias en múltiples sistemas, esquemas de percepción, pensamiento y acción, es decir, en diversas culturas» (p. 77). Esta definición implica que los profesores reconozcan que todo individuo pertenece a diversas culturas, y la necesidad de traer a la clase esa diversidad, fomentando tanto la pluralidad de contenidos como de métodos de transmisión, y promover la conciencia cultural y el análisis crítico.

Canale (1996), profundizó en cuanto a las directrices para un enfoque comunicativo con el fin de lograr un enfoque integrador en el que el objetivo principal es preparar y animar a los aprendientes a explotar de una forma óptima su limitada competencia comunicativa de la segunda lengua con el fin de participar en situaciones reales de comunicación. Las personas utilizan las competencias que desarrollan en distintos contextos y bajo distintas 
condiciones y restricciones con el fin de realizar actividades comunicativas que conllevan diferentes procesos para producir y comprender textos relacionados con determinados temas en ámbitos específicos, utilizando las estrategias que parecen más apropiadas para llevar a cabo las acciones que han de realizar. Es decir que, para que una persona que aprende una lengua extranjera desarrolle determinadas competencias en este sentido deben tenerse en cuenta aspectos tales como el contexto, el texto y el ámbito.

El concepto de competencia comunicativa se ha ido reelaborando desde su primera formulación (Hymes, 1971), con los aportes de diversos investigadores hasta llegar a constituir un complejo de subcompetencias. En uno de los modelos más extendidos, el de Michael Canale y Merril Swain (1980), después ampliado por Canale (1983), se describen las siguientes: a) competencia gramatical: supone el dominio del código lingüístico, es decir, de la gramática, del sistema fonológico y del léxico, y b) competencia sociolingüística: tiene que ver con el conocimiento de las propiedades de los enunciados en relación con el contexto social y la situación de comunicación en los que se producen (la información compartida entre los interlocutores, las intenciones comunicativas de la interacción, entre otros).

La competencia sociolingüística, hace referencia a la capacidad de una persona para producir y entender adecuadamente expresiones lingüísticas en diferentes contextos de realidad, en los que se dan variaciones tales como la situación de los participantes, su intención, el acto comunicativo y las normas que lo regulan.

No se puede desconocer que el contexto es una gran limitante para aquel que quiera enseñar y aprender inglés en Colombia, debido a la relativa necesidad de hacer uso de esta lengua en situaciones reales de comunicación y supervivencia. Al respecto, Ordóñez (2010: 3), señala que el principal problema para el aprendizaje de lenguas extranjeras que se enfrenta en contextos como el colombiano, es que el contexto sociolingüístico es mayoritariamente monolingüe en español. Esto quiere decir que los niños, niñas y jóvenes escolares no necesitan usar el inglés para funcionar en su vida diaria, lo cual hace especialmente difícil para ellos aprenderlo, aunque las políticas educativas nacionales establezcan que lo que debe suceder en los colegios respecto al aprendizaje del inglés está relacionado con la adquisición de la competencia discursiva, la cual se refiere al conocimiento de las relaciones entre los diferentes elementos de un mensaje y al dominio de las normas de combinación de dichos elementos de acuerdo con los diferentes tipos de textos. Además, la competencia estratégica, que hace referencia al dominio de las estrategias de comunicación verbal y no verbal para controlar la comunicación, para reforzar la eficacia de la misma o para compensar el insuficiente dominio de otras competencias.

En el enfoque comunicativo se promulga un tipo de enseñanza centrada en el alumno, en sus necesidades tanto comunicativas como de aprendizaje. Esto 
supone una pérdida de protagonismo por parte del profesor y de los programas, en favor de una mayor autonomía de los estudiantes y, por lo tanto, de una mayor responsabilidad en la toma de decisiones sobre su propio proceso de aprendizaje. De esta manera, el énfasis del enfoque comunicativo recae por lo tanto sobre los procesos implicados en el uso del lenguaje, es decir, sobre el estudio de los significados, de su expresión, comprensión y negociación durante las interacciones.

Teniendo en cuenta los Estándares Básicos de Competencias en Lenguas Extranjeras: Inglés, expuestos por el Ministerio de Educación Nacional de Colombia «MEN»(2006: 5), la competencia comunicativa en inglés incluye tres competencias: Lingüística, Pragmática y Sociolingüística.

La Competencia Lingüística, se refiere al conocimiento de los recursos formales de la lengua como sistema y a la capacidad para utilizarlos en la formulación de mensajes bien formados y significativos. Incluye los conocimientos y las destrezas léxicas, fonológicas, sintácticas y ortográficas, entre otras. Esta competencia implica, no sólo el manejo teórico de conceptos gramaticales, ortográficos o semánticos, sino su aplicación en diversas situaciones. Por ejemplo, hacer asociaciones para usar el vocabulario conocido en otro contexto o aplicar las reglas gramaticales aprendidas en la construcción de nuevos mensajes.

La Competencia Pragmática se relaciona con el uso funcional de los recursos lingüísticos y comprende, en primer lugar, una competencia discursiva que se refiere a la capacidad de organizar las oraciones en secuencias para producir fragmentos textuales. En segundo lugar, implica una competencia funcional para conocer, tanto las formas lingüísticas y sus funciones, como el modo en que se encadenan unas con otras en situaciones comunicativas reales (MEN, 2006: 5).

La Competencia Sociolingüística, se refiere al conocimiento de las condiciones sociales y culturales que están implícitas en el uso de la lengua. Por ejemplo, se emplea para manejar normas de cortesía y otras reglas que ordenan las relaciones entre generaciones, géneros, clases y grupos sociales. También se maneja al entrar en contacto con expresiones de la sabiduría popular o con las diferencias de registro, de dialecto y de acento.

De esta manera, la competencia comunicativa no se puede trabajar aisladamente pues implica un saber/hacer flexible, que se actualiza en contextos significativos y que supone la capacidad para usar los conocimientos acerca de la lengua en diversas situaciones, tanto dentro como fuera de la vida escolar. Desarrollar esas habilidades y saberes permite a los estudiantes ampliar sus conocimientos sobre el mundo, explorar sus habilidades sociales y conocer los aspectos culturales propios de la lengua que se aprende. 


\section{Estrategias pedagógicas}

Las estrategias de aprendizaje consisten en un procedimiento o conjunto de pasos o habilidades que un estudiante adquiere y emplea de forma intencional como instrumento flexible para aprender significativamente y solucionar problemas y demandas académicas. Por su parte, las estrategias de enseñanza son todas aquellas ayudas planteadas por el docente, que se proporcionan al estudiante para facilitar un procesamiento más profundo de la información (Díaz y Hernández, 1999).

Las estrategias de aprendizaje han sido consideradas especialmente importantes para el aprendizaje del idioma, puesto que constituyen herramientas para el estudiante en el involucramiento activo y autodirección del aprendizaje, lo que es esencial para el desarrollo de la competencia comunicativa (Oxford, 1990; Richards y Lockhart, 1994; Monereo, 2004; Casar y Hernández, 2000). Las estrategias que se pueden utilizar en el aula son útiles siempre y cuando se trate de una actividad del profesor, una actividad del alumno, una organización del trabajo a desarrollar, una organización del espacio y del tiempo, de materiales. Una estrategia educativa es «un plan para lograr los objetivos de aprendizaje, e implica métodos, medios y técnicas a través de los cuales se asegura que el alumno logrará realmente sus objetivos y que la estrategia elegida determinará de alguna forma el conjunto de objetivos a conseguir y, en general, toda la práctica educativa» (Salina, 2004: 189).

Algunas de las estrategias utilizadas en la presente investigación fueron el aprendizaje colaborativo y/o cooperativo, el cuál fue provechoso para los estudiantes de grado octavo que estaban aprendiendo un segundo idioma a través de la web Rosseta Stone. Se evidenció el desarrollo de actividades de aprendizaje colaborativo y cooperativo, las cuáles estimularon la interacción entre pares, ayudándoles al desarrollo del lenguaje y al aprendizaje de conceptos y contenidos. Los estudiantes aprendieron a expresarse con mayor confianza cuando trabajaron en equipos reducidos, de igual manera, contribuyeron al desarrollo de sus habilidades y destrezas sociales mediante el trabajo por parejas y en grupos, potenciando así su dimensión social y cooperativa. Además de «captar» vocabulario, los estudiantes se beneficiaron al observar cómo sus pares aprendían y resolvían problemas.

\section{Resultados}

El proceso investigativo permite visibilizar y evidenciar cómo la gran mayoría de estudiantes mejoraron su competencia comunicativa relacionada con el aprendizaje del inglés y se apropiaron de un nuevo vocabulario expuesto en diversos contextos comunicativos. De igual forma, los estudiantes participaron en forma activa, intercambiaron opiniones y consultaron algunas páginas 
dedicadas exclusivamente a temas relacionados con el proceso enseñanza aprendizaje de los idiomas, donde aparecen temas específicos de fonética, vocabulario, gramática, conversación, consulta de diccionarios y traductores, así como infinidad de aportes de tipo cultural.

Los estudiantes incorporaron y se apropiaron del ciberespacio en su proceso de aprendizaje del inglés. Por ejemplo, la docente los guio con algunas páginas interactivas relacionadas con el aprendizaje del mismo como fueron pumarosa, duolingo, la mansión del inglés, entre otras, permitiéndoles de esta manera fortalecer su competencia comunicativa en lengua extranjera. Así mismo, usaron algunos diccionarios bilingües on-line que permitieron enriquecer su léxico personal y además desarrollaron algunos test en la web que permitieron percibir sus debilidades y fortalezas en el idioma inglés como segunda lengua.

Muchas tareas se resolvieron por medio del trabajo colaborativo, y esto permitió que algunos estudiantes recibieran ayuda, diálogo permanente, socialización y retroalimentación de sus pares; reconociendo también el valor social que tiene el hecho de trabajar con los demás, en lugar de hacerlo en competencia con ellos. Los resultados de este tipo de tareas demuestran una vez más, que el aprendizaje que se produce muchas veces supera al que podría haber logrado cada alumno individualmente.

Los estudiantes interactuaron permanentemente con sus pares realizando un sinnúmero de actividades relacionadas con el aprendizaje del inglés como diálogos, comprensión de lecturas y test on-line. Igualmente diseñaron algunas presentaciones en Power Point con el fin de exponer algunas temáticas en inglés, agregándoles imágenes de la misma web. Utilizaron y se apropiaron del manejo de algunos programas como «sv recorder», para realizar grabaciones, Power Point Convertor, para pasar las diapositivas realizadas en Power Point a vídeo y realizaron diferentes trabajos aplicativos subiendo algunas actividades asignadas en clase, las cuales fueron expuestas en un blog, el cual se anexa en el enlace http://repositorioingles.wordpress.com/, evidenciando así el mejoramiento de las cuatro habilidades del Inglés «Listening, Speaking, Reading and Writting».

En este tipo de interactividad con la web, los estudiantes tuvieron la oportunidad de realizar actividades no solamente a nivel on-line sino también off-line, en las cuales se evidencia el desarrollo de las cuatro habilidades comunicativas del inglés (Listening, Speaking, Reading and Writting) y por ende el trabajo colaborativo.

En relación con la habilidad de Listening los estudiantes ingresaron a varios enlaces inmersos en el mundo del ciberespacio y en forma autónoma observaron diversos videos en inglés, escucharon diálogos, canciones, escucharon las opiniones de los demás e interactuaron permanentemente junto con la docente, haciendo la respectiva retroalimentación tanto de las actividades on-line como off-line. 
En cuanto a la habilidad de Reading, los estudiantes hicieron lecturas de textos cortos y largos, todos enfocados a la comprensión de los mismos. Luego leyeron las opiniones de los demás, realizando las observaciones pertinentes junto con la docente.

Al respecto de la habilidad de Writing, los estudiantes fortalecieron su vocabulario usado en diversos contextos, permitiendo que ellos escribieran oraciones y párrafos cortos en inglés, así como articulando su aprendizaje del inglés con el uso del ciberespacio. Igualmente, se establecieron algunas normas y protocolos de clase que llevaron a cabo los estudiantes como fueron: contribución y dedicación a las tareas, ayuda mutua, compartieron experiencias, resolvieron problemas, dieron y aceptaron opiniones de sus pares.

Teniendo en cuenta los resultados planteados se desarrollaron estrategias de aprendizaje autónomo y cooperativo tales como la planificación, el repaso, la búsqueda, la selección y organización de la información, combinando de forma armónica el trabajo individual con el trabajo por parejas y en grupo.

El análisis efectuado refleja la necesidad de transformar los procesos de formación en relación con el aprendizaje del inglés, a partir de estrategias pedagógicas que propicien un óptimo aprovechamiento de la diversidad de recursos tecnológicos que favorezcan la continua colaboración y cooperación entre los sujetos participantes en estos entornos, tendientes a disminuir las falencias encontradas relacionadas con la adquisición de una segunda lengua, en este caso del inglés, especialmente en las habilidades de Speaking y Listening.

\section{Conclusiones}

El aprendizaje del inglés a través de plataformas interactivas es un campo fértil para futuras investigaciones. En la presente investigación se pudo constatar que el aprendizaje a través de la web implica mucha responsabilidad no sólo de parte del estudiante sino del docente-tutor. Los beneficios pueden ser variados, pero sólo si existe una planificación previa del diseño de soporte y apoyo efectivo a las actividades a nivel on-line y off-line que se proponen, con un monitoreo constante de los avances para identificar carencias y hacer mejoras; así como evaluaciones y tareas permanentes que promuevan e integren el desarrollo de las cuatro habilidades comunicativas del inglés serán aspectos esenciales para que nuestros estudiantes logren la competencia comunicativa en el idioma inglés de manera exitosa.

El principal aporte de esta investigación al campo de la pedagogía y teniendo en cuenta los resultados obtenidos en la misma, se relaciona con el uso del ciberespacio y de las herramientas de la web, como un aporte significativo en el mejoramiento de las cuatro habilidades comunicativas del inglés (Listening, 
Speaking, Reading and Writing), fortaleciendo y mejorando el proceso de aprendizaje del inglés de los estudiantes en forma autónoma e interactiva.

Los procesos de interactividad con la web Rosseta Stone contribuyeron significativamente en el proceso de aprendizaje de los estudiantes de grado octavo del Colegio León de Greiff, institución de carácter oficial y rural, ubicada en el Municipio de Aguazul, ya que aportaron al mejoramiento de las cuatro habilidades comunicativas de idioma inglés (Listening, Speaking, Reading and Writing).

La web Rosseta Stone puede considerarse una herramienta enriquecedora y significativa en el mejoramiento de la competencia comunicativa del inglés, siempre y cuando los procesos de interactividad llevados a cabo sean pertinentes, dinámicos y continuos. Se evidenció que la web es un espacio que le permite al estudiante el aprender a aprender de manera dinámica, participativa y atractiva.

En relación con los procesos de interactividad que realizaron los estudiantes con dicha web, se evidenció el desarrollo de actividades significativas. Inicialmente cabe resaltar la habilidad de Listening: los estudiantes tuvieron la oportunidad de escuchar palabras, frases, diálogos y canciones, así como ver vídeos en diversos contextos comunicativos. De igual manera, estuvieron siempre dispuestos a escuchar los aportes e intercambio de las experiencias de cada uno de los compañeros y las instrucciones permanentes de la docente, enriqueciendo de esta manera su habilidad de escucha.

Al respecto de la habilidad de Speaking los estudiantes expresaron sus opiniones y compartieron información con sus pares, contando historietas cortas a través de frisos e interactuando de forma permanente en forma oral, mejorando su pronunciación y por ende su habilidad de habla. En cuanto a la habilidad de Reading, los estudiantes leyeron varias palabras y frases en diversos contextos comunicativos, afianzando así su lectura, comprensión de texto y adquisición de nuevo vocabulario. Para finalizar, en la habilidad de Writing, los estudiantes desarrollaron varias actividades on-line y off-line, donde se evidenció el mejoramiento en los aspectos gramatical, semántico y sintáctico, desarrollando así su habilidad de escritura.

Es importante destacar que los estudiantes junto con la docente hicieron retroalimentación de cada una de las actividades realizadas a nivel on-line y off-line, concluyendo que es necesario el refuerzo en el campo gramatical y en la pronunciación de los estudiantes. El trabajo colaborativo entre pares fortalece los procesos de resolución de problemas, los valores, las actitudes y el comportamiento personal de los estudiantes.

El ciberespacio es una herramienta pedagógica que potencializa los procesos de aprendizaje del inglés, siempre y cuando exista la guía de un tutor, la 
responsabilidad de los estudiantes y la motivación personal para aprender en forma autónoma y de manera colaborativa. De esta manera, el aprendizaje colaborativo y cooperativo dota al estudiante no solo de elementos necesarios para que pueda interactuar con sus pares y aprender de esta interacción, sino que también lo prepara para tener un buen desempeño en sus procesos de enseñanza y aprendizaje.

Así mismo, a través de esta investigación se evidenció que los estudiantes incorporaron algunas estrategias didácticas y pedagógicas entre las que cabe mencionar juego de roles, trabajo colaborativo en la realización de actividades a nivel on-line y off.line con su respectiva retroalimentación, con las cuáles potencializaron sus conocimientos relacionados con el aprendizaje del idioma inglés.

De acuerdo con los aportes de Levy, se constató que el ciberespacio constituye un espacio de cultura colectiva donde los estudiantes se apropiaron de valores y enriquecieron sus conocimientos a través de las experiencias compartidas.

El aprendizaje del idioma inglés depende de la interacción con otros. En este sentido, el aprendizaje colaborativo o cooperativo ofrece a los estudiantes la oportunidad de interactuar en la lengua al resolver una tarea que requiere el uso comunicativo del idioma para alcanzar un objetivo. Esta tarea se desarrolla en grupos cuyos integrantes deben negociar significados, trabajar en forma autónoma, asumir roles dentro del grupo y responsabilizarse por su trabajo. El aprendizaje de una segunda lengua se facilita cuando los estudiantes tienen la oportunidad de participar en interacciones en las que deben comunicar y negociar significados. 
Estrategias pedagógicas para el aprendizaje del inglés con el uso de la herramienta Rosseta Stone por parte de los estudiantes de grado octavo del Colegio León de Greiff. Artículo producto de la investigación.

\section{Referencias bibliográficas}

Acosta, M. 2004. Tecnología y nuevas tecnologias de comunicación: reflexiones desde una perspectiva histórica. en: LatinEduca. Disponible en: Tecnología y nuevas tecnologías de comunicación: reflexiones desde una perspectiva histórica. http:// www.ateneonline.net/datos/92_03_Acosta.pdf

Ausubel, D. 1976. Teoría del aprendizaje significativo.

Bonilla, E. y Rodríguez, P. 1997. Más allá del dilema de los métodos. La investigación en las ciencias sociales. Bogotá: Norma.

Bustos, A. \& Coll S. C. 2010. Los entorno virtuales como espacio de enseñanza y aprendizaje: una perspectiva psicoeducativa para su caracterización y análisis. Revista Mexicana de Investigación Educativa, 15 ( 44): 163-184. Consejo Mexicano de Investigación Educativa. México. Disponible en: http://www.redalyc.org/articulo. oa?id=14012513009

Cabero, J. 1996. Las posibilidades de las nuevas tecnologías de la información y la comunicación para el desafio de la educación de personas adultas, en: Agenda Académica. Disponible en: http://edutec.rediris.es/documentos/1996/agendave.htm

Cabrero, J. 2007. Nuevas tecnologías aplicadas a la educación. Madrid: Mc. Graw Hill.

Cárdenas, P. 1997. Hacia una pedagogía integral del lenguaje. Disponible en; http:// www.pedagogica.edu.co/storage/folios/articulos/folo7_06art.pdf

Castells, M. 1995. La ciudad informacional. Madrid: Alianza Editorial.

Charaudeau, P. 1986. Análisis del discurso y sus implicaciones pedagógicas. Cali: Univalle y oficina lingüística y audiovisual -servicio cultural de la embajada de Francia.

Chen, Y. 2001. Dimensions of transactional distance in the world wide web learning environment: a factor analysis. British Journal of Educational Technology, 32 (4): 459470.

Coll, C. 2004. Psicología de la educación y prácticas educativas mediadas por las tecnologías de la información y la comunicación. Una mirada constructivista. Facultad de Psicología. Universidad de Barcelona. Disponible en: http://www.virtualeduca.org/ ifd/pdf/cesar-coll-separata.pdf

Daiute, C. 1998. Points of view in children's writing. Language Arts, 75: 138-149.

Dam, L. 1990. Learner autonomy in practice. Autonomy in Language Learning. London: Gathercole. 
Díaz, B. \& Hernández, G. 1999. Estrategias docentes para un aprendizaje significativo: una interpretación constructivista. México: McGraw Hill.

Fandiño, Y., Bermúdez, J. \& Lugo-V, E. 2012. Retos del programa nacional de bilingüismo. Colombia Bilingüe. Educ. . 15 (3): 363-381.

García, F. 2004 ¿Educar en el ciberespacio o educar para el ciberespacio? Disponible en: http://www.ugr.es/ sevimeco/revistaeticanet/Numero3/Articulos/Formateados/ Educarenel ciberespacio[1].pdf. Granada (España). Año II Número 3.

García, S. 2009. Adquisición de nuevo vocabulario en inglés. Revista Digital Innovación y Experiencias Educativas. 18: 1-13. Disponible en: http://revistavirtual.ucn.edu.co/ index.php/ RevistaUCN/article/viewFile/408/805

González, L. \& Flórez. 2000. La Educación Superior a Distancia en Colombia. Bogotá: ICFES.

Halliday, M. 1978. El lenguaje como semiótica social. La interpretación social del lenguaje y del significado, México D.F.: Fondo de Cultura Económica.

Hernández, S. 2008. El modelo constructivista con las nuevas tecnologías, aplicado en el proceso de aprendizaje. Revista de Universidad y Sociedad del Conocimiento. 5: (2) Disponible en http://www.uoc.edu/rusc/5/2/dt/esp/hernandez.html

Hymes, D. 1971. Acerca de la competencia comunicativa. Documentos básicos en la enseñanza de lenguas extranjeras. Madrid: Edelsa.

Holmberg, B. 1986. Educación a distancia. Situación y perspectivas. Buenos Aires: Kapelusz.

Kerckhove, D. 1999. Inteligencias en conexión: hacia una sociedad de la web, Barcelona: Gedisa.

Labañino, C. 2006. El software educativo. Módulo 1. Fundamentos de la investigación Educativa en Curso 4. Las Nuevas Tecnologías de Información y la Comunicación en la institución educativa para el inicio de la Maestría en Ciencias de la Educación. en CD Maestría en Ciencias de la Educación. Parte I. Soporte digital. Disponible en: http:// www.eumed.net/rev/ced/27/mrh.htm

Lévy, P. 2007. Cibercultura: la cultura de la sociedad digital. Barcelona: Anthropos.

Lucas, M. 2000. La nueva sociedad de la información: una perspectiva desde Silicon Valley. Madrid: Trotta. 
Estrategias pedagógicas para el aprendizaje del inglés con el uso de la herramienta Rosseta Stone por parte de los estudiantes de grado octavo del Colegio León de Greiff. Artículo producto de la investigación.

Marabotto, M. 2005. La calidad de la mediación didáctica en el marco de las tecnologías de la información y la comunicación. C\&P Comunicación y Pedagogía. Revista Nuevas Tecnologías y Recursos Didácticos, Nㅜ 204.

Márques, P 2000. Impacto de las TIC en la enseñanza universitaria. Disponible en: http://peremarques.pangea.org/ticuniv.htm

Martí, E. 2003. Representar el mundo externamente. Madrid: Aprendizaje.

Ministerio de Educación Naciona. 2005. Colombia bilingüe. Al Tablero, 37. Disponible en: http://www.mineducacion.gov.co/1621/article-97495.html

Ministerio de Educación Nacional. 2006. Estándares Básicos de Competencias en Lenguas Extranjeras: Inglés. Colombia.

Ministerio de Educación Nacional. 2010. Proyecto de Fortalecimiento al Desarrollo de Competencias en Lenguas Extranjeras. Disponible en: http://www.mineducacion. gov. co/1621/articles-327001_archivo_pdf_terminos_convocatoria.pdf

Nunan, D. 1991. Communicative tasks and the language curriculum, Tesol Quarterly. 25 (2).

Nuñez, M. s.f. Los micro ciber-juegos y las actividades interactivas como herramientas en la enseñanza complementada por Internet. Disponible en: http://www.uprm.edu/ ideal/juegos-web.pdf

OCDE 2002. Conocimientos y aptitudes para la vida. Primeros resultados del PISA 2000 de la OCDE. Madrid: Santillana; MEC.

Ordóñez, C. 2010. Educación para el bilingüismo en contexto monolingüe. Dos lenguas conectadas desde el currículo. Elia: Estudios de lingüistica inglesa aplicada, 10: 41-76. Disponible en: http://institucional.us.es/revistas/elia/10/5.Ordonez.pdf

Oxford, R. 1990. Language Learning Strategies: What Every Teacher Should Know. Boston, MA: Heinle \& Heinl.

Piaget, J.1978. La función simbólica de Piaget. Estudios de Psicología Genética. Buenos Aires: Emecé.

Piscitelli, A. 1995. Ciberculturas: en la era de las máquinas inteligentes. Buenos Aires: Paidós.

Praot, A. \& Mendoza, M. 2006. Opinión, conocimiento y uso de portales web para la enseñanza del inglés como lengua extranjera. Disponible en: http://www.scielo.org.ve/ scielo. php?pid=S16905152006000100004\&script=sci_arttext 
Rodríguez, E. 2009. Motivación factor clave en el proceso de enseñanza y aprendizaje del inglés en un entorno virtual. Tesis de Grado. Disponible en: http://www.umariana. edu.co/RevistaUnimar/index.php/revista-unimar-no-59/808-la-motivacion-factorclave-en-el-proceso-de-ensenanza-y-aprendizaje-del-ingles-en-un-entorno-virtual.

Sabina, P. Dovalí, G. \& Prieto, P. 2007. Memorias del III Foro Nacional de Estudios en Lenguas.

Salinas, J. 2004. Cuadernos de Pedagogía. Madrid: Sociedad Española de Pedagogía. Sallita.net aprendizaje virtual interactivo. http://sallita.educa.com.mx/

Sanhueza, E. 2005. El uso de internet en el aprendizaje de la pronunciación inglesa, una experiencia con estudiantes de ingeniería. Tesis de Grado de Maestría en Educación. (Santiago, Chile 2005). Disponible en: http://www.tesis.uchile.cl/tesis/uchile/ 2005/ sanhueza_e/sources/sanhueza_e.pdf

Sims, R. 1995. Interactivity: A Forgotten Art. Instructional Technology Research. Disponible en: http://www.researchgate.net/publication/222501756_Interactivity_A_ forgotten_art.

Taylor, S. \& Bodgan. 1986. Introducción a los métodos cualitativos de investigación. Buenos Aires: Paidós.

Vasilachis, I. 2006. La investigación cualitativa. Estrategias de investigación cualitativa. Barcelona: Gedisa.

Vygotsky, L. 1979. El desarrollo de los procesos psicológicos superiores. Barcelona: Crítica.

Watzlawick, P. 1991. Teoría de la comunicación humana. Barcelona: Herder. 